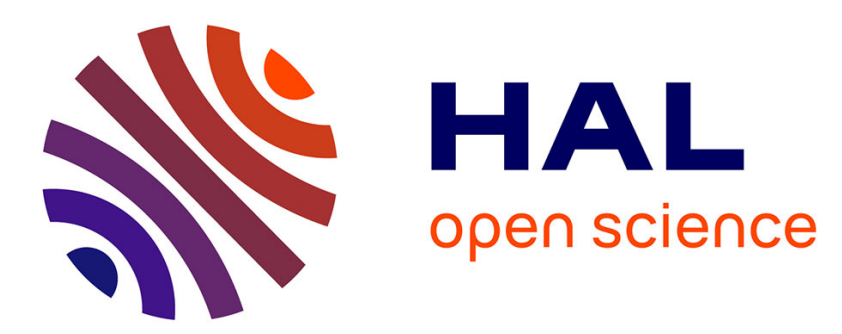

\title{
Automatic detection of microemboli by means of a synchronous linear prediction technique
}

Sébastien Ménigot, Latifa Dreibine, Nawal Meziati, Jean-Marc Girault

\section{To cite this version:}

Sébastien Ménigot, Latifa Dreibine, Nawal Meziati, Jean-Marc Girault. Automatic detection of microemboli by means of a synchronous linear prediction technique. Ultrasonics Symposium (IUS), 2009 IEEE International, Sep 2009, Rome, Italy. pp.2371 - 2374, 10.1109/ULTSYM.2009.5441701 . hal-00598463

\section{HAL Id: hal-00598463 https://hal.science/hal-00598463}

Submitted on 6 Jun 2011

HAL is a multi-disciplinary open access archive for the deposit and dissemination of scientific research documents, whether they are published or not. The documents may come from teaching and research institutions in France or abroad, or from public or private research centers.
L'archive ouverte pluridisciplinaire HAL, est destinée au dépôt et à la diffusion de documents scientifiques de niveau recherche, publiés ou non, émanant des établissements d'enseignement et de recherche français ou étrangers, des laboratoires publics ou privés. 


\title{
Automatic detection of microemboli by means of a synchronous linear prediction technique
}

\author{
Sébastien Ménigot ${ }^{1}$, Latifa Dreibine ${ }^{1}$, Nawal Meziati ${ }^{1}$ and Jean-Marc Girault ${ }^{1}$, Member, IEEE \\ ${ }^{1}$ Université François Rabelais de Tours, Tours, France \\ INSERM, U930, Tours, France \\ CNRS, ERL 3106, Tours, France
}

\begin{abstract}
Detection of microemboli is of great clinical importance to prevent cerebro-vascular events and to identify the causes of such events. As standard detection techniques implemented in the most commonly used systems cannot detect all of microemboli events whose energy is lower than the systolic energy, new techniques are proposed.

By assuming that the Doppler signal is cyclostationary, we hypothesize that energy is statistically periodic. Furthermore, we hypothesis that embolic signals are unpredictable. Hence, the joint use of synchronous and linear prediction techniques could detect very small microemboli. If we periodically take and compare the values of the energy of the prediction error (or autoregressive parameters) at different time points in the cardiac cycle, we can therefore detect the presence of non-periodic events such as microemboli.

In our study, we tested and compared our new technique to the standard technique (Fourier) using simulated and in vivo signals from patients with stenosis of high degrees of severity. From simulations, the standard automatic technique detected $60 \%$ of microemboli detected by our gold standard technique (audible detection and sonogram visualization) whereas the synchronous linear prediction technique detects $97 \%$ (the false alarm rate being set at $0 \%$ ). From clinic examinations, the standard automatic technique only detects $67 \%$ of microemboli detected by our gold standard technique whereas the synchronous linear prediction technique detected $100 \%$.

This study demonstrates that our new technique detects microemboli up to now not identified by classical methods. Large microemboli are all detected, but small microemboli are only detected with our new technique. This latter technique opens up new prospects to detect small emboli, despite the need for further studies to incorporate "on line" technique.
\end{abstract}

Index Terms-Microemboli, synchronous detection, linear prediction.

\section{INTRODUCTION}

$\mathbf{C}$ EREBRAL vascular accidents and particularly cerebral embolisms represent more than two thirds of all ischemic

(c) 20009 IEEE. Reprinted, with permission, from S. Ménigot, L. Dreibine, N. Meziati and J.-M. Girault, Automatic detection of microemboli by means of a synchronous linear prediction technique, 2009 IEEE International Ultrasonics Symposium (IUS), 20-23 Sept. 2009.

Digital Object Identifier: 10.1109/ULTSYM.2009.5441701

This material is posted here with permission of the IEEE. Such permission of the IEEE does not in any way imply IEEE endorsement of any of the Université François Rabelais de Tours' products or services. Internal or personal use of this material is permitted. However, permission to reprint/republish this material for advertising or promotional purposes or for creating new collective works for resale or redistribution must be obtained from the IEEE by writing to pubs-permissions@ieee.org. strokes. Indeed, several insoluble bodies (fat, red cell aggregation, clots...) foreign to blood composition, called emboli, can move into intracranial arteries and can even block them. The detection of microemboli (small size emboli) is important for several reasons such as preventing cerebrovascular accidents, finding the cause of embolism and validating the effectiveness of treatment.

The TransCranial Doppler ultrasound (TCD) system is the most used technique in detecting and counting emboli[1]. This standard technique seems to be sufficient to detect most of microembolic events. Nevertheless during clinical examinations, the medical profession sometimes observes microembolic signatures not detected by the system.

The embolic Doppler signature is an unpredicted high intensity transient signal (HITS) superimposed on the Doppler signal backscattered by the blood, the embolus detection must be therefore based on the energy.

By assuming that the Doppler signal is cyclostationary [2], we suppose that the energy is statistically periodic. Furthermore, by assuming that HITS are unforcasted events, we implicitly suppose that linear prediction techniques are unable to predict the presence of a microembolus. The joint use of synchronous and linear prediction techniques could be the foundation of new techniques allowing the detection of very small microembolus.

By taking and comparing periodically the values of the prediction error and autoregressive (AR) parameters (at different time points in the cardiac cycle), it is possible to detect the presence of non-periodic events such as microemboli.

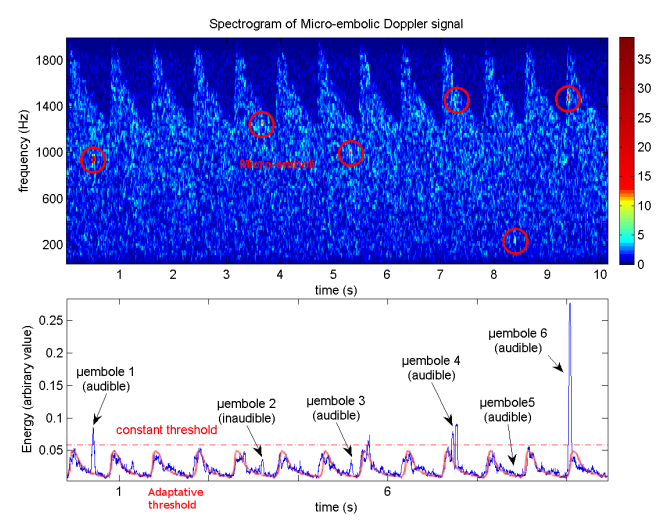

Figure 1. Embolus detection with a constant threshold : spectrogram (top) and time-varying energy (bottom) 


\section{MATERIALS AND METHODS}

\section{A. Materials}

The TCD system measures the velocity of blood flow through the brain's arteries with a pulsed Doppler probe. Because the skull bones reduce the transmit ultrasound (US) power, the recording is performed in the temporal region. The TCD consists in transmitting, by means of a transducer, an US wave with frequency $f_{0}$ at $2 \mathrm{MHz}$, to a selected area of a cerebral artery. The TCD systems that we used was the $\mathrm{Waki}^{\mathrm{TM}}$ (Atys Medical, Soucieu en Jarrest, France). The pulse repetition frequency (PRF) was of $6.4 \mathrm{kHz}$ and the US power was of $150 \mathrm{~mW} / \mathrm{cm}^{-2}$. The Doppler signals are sampled with a frequency of $10 \mathrm{kHz}$.

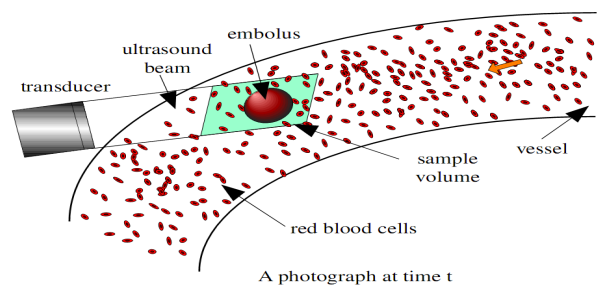

Figure 2. Sketch of a solid embolus in the sample volume

The Doppler embolus signal was modeled by using the method proposed by Girault et al.[3]. The most of the instantaneous energy of each embolus was chosen in order to be lower than the highest level of the background Doppler signal which has a 5 minutes length. As our main objective was to detect very small embolus signatures from 9 different Doppler signals.

The in vivo Doppler signals from patients with stenosis of degrees IV of severity have been analysed.

The position of the Doppler embolus signal has been known in order to constitute our gold standard. Each Doppler signal has been listened and analysed from its sonogram.

\section{B. Protocole}

Microembolus signals are detected by a binary test. If the decision information $D I(t)$ which corresponds to the instantaneous energy is greater than the threshold $\lambda$ then an embolus is detected (hypothesis $H_{1}$ ), otherwise no embolus is detected (hypothesis $H_{0}$ ). This formulation can be expressed in equation 1

$$
D I(t) \underset{H_{0}}{\lessgtr} \lambda
$$

Each method requests a preliminary step where statistical thresholds are computed. During the first minute, the statistic parameters are collected in order to establish the threshold $\lambda$. After that, the threshold is updated from the statistic parameters of the last minute. In the case of the standard detection, the instantaneous energy was computed with a Hamming slidding window (128 samples). As the instantaneous energy follows a Gaussian law, the constant threshold can be set to $\lambda=\mu+5 \sigma$ where $\mu$ is the mean and $\sigma$ is the standard deviation.

In the case of the synchronous detector, the algorithm is the following :
- From an examination, we compute first the energy over a Hamming slidding window of 128 samples and second the maximal cardiac cyclic period.

- Then all the cardiac cycles are extracted at each beginning of the systole phase. In order to establish some statistic parameters from data having the same number of samples, we resample and interpolate the whole cardiac cycles.

\section{C. standard method}

1) standard energy estimator: The standard energy estimator implemented in the most commonly used systems is based on the spectrogram or Short Time Fourier Transform (STFT). The basic idea is to find the power spectrum density $P(t, f)$ (eqn 2) of the signal at time $t$ by analyzing a small segment of the signal around it.

$$
P(t, f)=\left|\int_{-\infty}^{+\infty} x(\tau) g^{*}(t-\tau) \mathrm{e}^{-j 2 \pi f \tau} \mathrm{d} \tau\right|^{2}
$$

where $x(t)$ represents the analysed Doppler signal. The signal is multiplied by a window function $g$ in order to reduce the random effects resulting from the computation of $P(t, f)$. The superscript asterisk denotes complex conjugation. While a long analysis window may improve frequency resolution, it compromises the assumption of stationarity within the window. The Doppler signal can be regarded as locally stationary over a duration lower than $10 \mathrm{~ms}$.

The decision information (DI) is the same as in commercial devices, i.e. it is $D I_{1}$ the mean of the power spectrum or $D I_{2}$ the maximum power spectrum :

$$
D I_{1}(t)=\left.\overline{P(t, f)}\right|_{f} \text { or } D I_{2}(t)=\left.\max (P(t, f))\right|_{f}
$$

Though these DI are time-varying all commercial devices are based on a constant threshold.

2) standard energy detector: The DI on which is performed the microembolus detection is the instantaneous energy.

1) Due to both the random nature of the Doppler signal and the limit central theorem, energy can be approximated by a Gaussian random variable $N\left(\mu, \sigma^{2}\right)$ where $\mu$ and $\sigma$ denote respectively the mean and the standard deviation of the energy. Note this case as the hypothesis $H_{0}$.

2) If we hypothesis that a patient provides only one type of emboli whose size is constant (for example $100 \mu \mathrm{m}$ ), then the embolic energetic information can be viewed as a Gaussian random variable $N\left(\mu_{e}, \sigma_{e}^{2}\right)$, where $\mu_{e}$ and $\sigma_{e}$ respectively denote the mean and the standard deviation of the energy in presence of embolus. This case, noted hypothesis $H_{1}$, is not a realistic hypothesis but largely contributes to simplify the reasoning leading to the decision defined by the equation (1).

This means that $\mu_{e}$ is also a random variable and consequently that the threshold has to be chosen empirically or statistically. The value of this threshold $\lambda$ was set just above the highest detected energy of the observed Doppler signal without embolus in the preliminary setting phase at 1 minute. The time position of this maximum energy always happens during the systole phase and its value largely depends on 
the patient but also of the duration of the examination. In order to have a weak false alarm probability, the threshold can be chosen just above the highest intra-individual energy, i.e. during the entire examination. However, the price to pay is that this threshold choice forbids all detections of microemboli events whose energy would be lower than this maximum value, i.e. during the systolic phase.

Most of the time, for a manual detection this empirical threshold is set around $3 \mathrm{~dB}$ above the maximal energy in order first to reduce the false alarm probability and secondly to take into account the inter individual variability. In contrast, for an automatic detection it is set between 3 to $9 \mathrm{~dB}$ and it depends on the TCD system calibration [4].

\section{Synchronous linear prediction technique}

1) Autoregressive modeling: Unlike used methods for embolus detection, parametric autoregressive (AR) modeling consists of working on a model of the signal and not directly on the signal. This filter is a linear combination of the previous samples (regressive) of the output itself (auto).

Considering a discrete time complex Doppler signal $x(n)=$ $x\left(t=n T_{e}\right)$ sampled at frequency $1 / T_{e}$ and assuming that it is the output of a p-order AR model, the signal can be expressed by the equation (4).

$$
x(n)=-\sum_{i=1}^{p} a_{i}(n) x(n-i)+\eta(n)
$$

where $a_{i}(n)$ are complex coefficients defining the AR model, $p$ is order of the model (number of coefficients) and $\eta(n)$ is a complex white noise. An order test shows that $p=2$ may be sufficient. For convenience, the previous equation is expressed in matrix form (eqn 5).

$$
x(n)=\varphi^{T}(n) \theta(n)+\eta(n)
$$

where

$$
\begin{gathered}
\varphi_{n}^{T}=[-x(n-1),-x(n-2)] \\
\theta=\left[a_{1}, a_{2}\right]^{T}
\end{gathered}
$$

The vector $\hat{\theta}(n)$ which is an estimate of the vector $\theta(n)$ was obtained by an AR process from the signal $x$. This estimation can be performed using the Recursive Least Squares (RLS) algorithm to minimize the prediction error $\varepsilon(n)$.

$$
\varepsilon(n)=x(n)-\hat{x}(n)
$$

The time-variant algorithm is accomplished by updating the previously evaluated parameter using the prediction error and weighting by means of a forgetting factor. In fact, using a forgetting factor is equivalent to the introduction of an exponential window. The length of this window indicates which of the last samples are in fact taken into account to compute AR parameters. For example, if the forgetting factor is equal to 0.95 , the last 20 samples are mainly accounted for in the window, whereas if it is equal to 0.99 the last 100 samples are taken into account. This adaptive least-squares method evaluates the parameters by minimizing the cost function (eqn 7 )

$$
J_{k}=\sum_{n=1}^{k} \lambda^{k-n}(x(n)-\hat{x}(n))^{2}
$$

where in practice $0.80<\lambda<1$ and $k$ is the index of the last sample considered.

The recursive adaptive algorithm is given by [3] :

$$
\begin{gathered}
P_{n}=\frac{P_{n-1}}{\lambda}\left(1-\frac{P_{n-1} \phi_{n} \phi_{n}^{T} P P n-1}{\lambda+\phi_{n}^{T} P_{n-1} \phi_{n}}\right) \\
\varepsilon_{n}=x_{n}-\phi_{n}^{T} \hat{\theta}_{n-1} \\
\hat{\theta}_{n}=\hat{\theta}_{n-1}+P_{n} \theta_{n} \varepsilon_{n}
\end{gathered}
$$

where $\hat{\theta}$ is estimated parameters vector, $P_{n}$ gain, $\phi_{n}$ observation vector and $\varepsilon_{n}$ prediction error.

The prediction error tends in probability to white noise, when the number of observation data extends to infinity. Since the autocorrelation function (AF) of a white noise equals zero at any lag, except for the initial lag $(n=0)$, the AF of prediction error therefore provides interesting Decision Information (DI), i.e. information containing the embolus signature for this parametric method. Indeed, when an embolus crosses the sample volume, the prediction error will no longer be a white noise, and its AF at lag 1 (for example) will no longer be zero. The AF can be expressed by :

$$
\Gamma_{\varepsilon \varepsilon}(n)=\sum_{m=-\infty}^{+\infty} \varepsilon(m) \varepsilon^{*}(m-n)
$$

Note that $\Gamma_{\varepsilon \varepsilon}$ will be almost zero for a normal Doppler signal and the presence of an embolus will be characterized by an abrupt change. The monitoring of the AF which seems to be a valuable $D I$ constitutes the novel $D I$

$$
D I=\left\|\Gamma_{\varepsilon \varepsilon}(n)\right\|
$$

2) Synchronous energy detector: The synchronous detection is a detection synchronized with the cardiac cycle. This synchronization is very important because it detects low energy events during the cardiac cycle. The specificity of this detector is that the threshold is time-varying [5]. In this case, the comparison of the instantaneous $D I(t)$ (energy) with a time-varying threshold $\lambda(t)$ leads to the decision :

$$
D I(t) \underset{H_{0}}{\lessgtr} \lambda(t)
$$

As it is previously mentioned $\mathrm{DI}(\mathrm{t})$ is statically periodic involving the adaptive threshold $\lambda(t)$ periodically time-varying.

In this case, as an embolus is a random event, it is possible to detect the presence of microemboli. From this energetic information, the "instantaneous" mean $\mu(t)$ and standard deviation $\sigma(t)$ of the cyclic energy have been computed. In order to have a weak probability of false alarm, it seems reasonable to choose an adaptive threshold defined by the equation 11 .

$$
\lambda(t)=\mu(t)+\beta \sigma(t)
$$

where $\beta$ is an empiric constant fixed to 3.8 . 

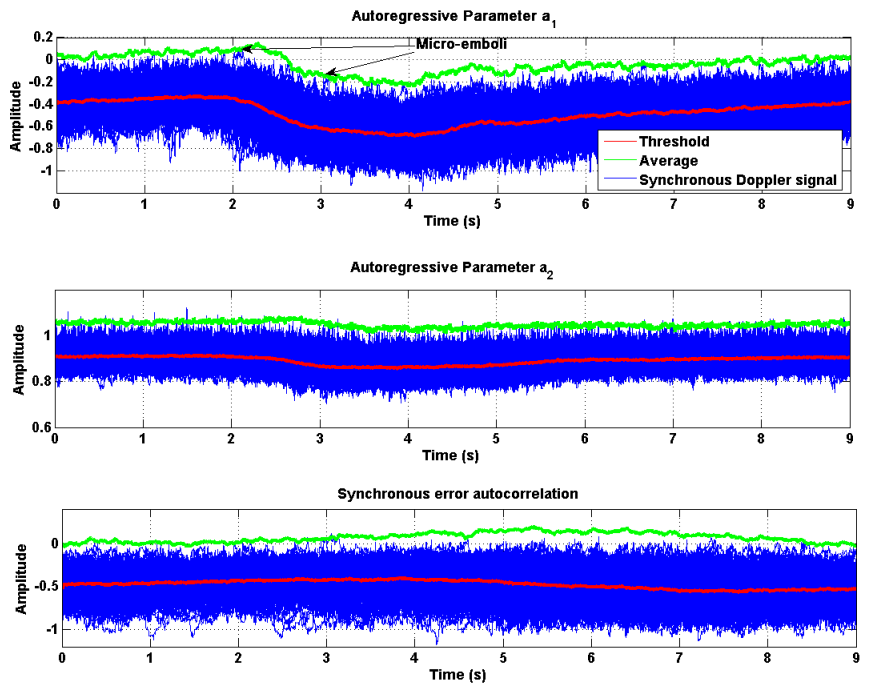

Figure 3. Synchronous AR parameters (top and middle) and synchronous error autocorrelation (bottomfolith their thaphardrying threstold

\begin{tabular}{lllll} 
& standard & & $a_{1}$ & error \\
\hline $\begin{array}{l}\text { embolus } \\
\text { detection }(\%)\end{array}$ & 100 & 100 & 100 & 100 \\
\hline $\begin{array}{l}\text { false alarm } \\
\text { rate }(\%)\end{array}$ & 0 & 32.37 & 7.49 & 5.80 \\
\hline
\end{tabular}

Table I

COMPARISON WITH STANDARD TECHNIQUE AND SYNCHRONOUS LINEAR PREDICTION TECHNIQUE(SLP) FOR $a_{1}$ PARAMETER AND THE AUTOCORRELATED ERROR FROM 9 SIMULATED SIGNALS

\section{RESUlTS \& Discussions}

\section{A. Simulation comparison}

An ideal detector is a technique which gives $100 \%$ of detection and $0 \%$ of false alarm. In this section, the threshold was set to give a detection rate of $100 \%$. The standard technique have detected $32 \%$ of false alarm for a detection rate of $100 \%$ compared with the gold standard. The new synchronous linear prediction technique (SLP) can reach a $5.8 \%$ false alarm rate with the autocorrelated error. The detector based on the error autocorrelation gives the best results because it includes the AR parameters $a_{1}$ and $a_{2}$ of AR modeling into account. Note that for synchronous detector the threshold is no more constant but rather time-varying. Note also that unlike synchronous AR parameters error autocorrelation seems to be not time-varyin. In case of the simulated signals, there exists inaudible events whose presence in the spectrogram is ambiguous. Our detectors allow us to detect such events.

\section{B. In vivo signal comparison}

In this section, the threshold was set to give a false alarm rate of $0 \%$. From clinic examinations, the standard automatic

\begin{tabular}{llll}
\hline & $\begin{array}{l}\text { Gold } \\
\text { standard }\end{array}$ & standard & SLP \\
\hline $\begin{array}{l}\text { embolus } \\
\text { detection }(\%)\end{array}$ & 100 & 67 & 100 \\
\hline $\begin{array}{l}\text { false alarm } \\
\text { detection }(\%)\end{array}$ & 0 & 0 & 0 \\
\hline \multicolumn{5}{c}{ Table II }
\end{tabular}

COMPARISON WITH STANDARD TECHNIQUE AND SYNCHRONOUS LINEAR PREDICTION TECHNIQUE (SLP) FROM in vivo SIGNALS technique only detects $67 \%$ microemboli compared with the gold standard. The new synchronous linear prediction technique (SLP) reaches the ideal detector with detection rate of $100 \%$.

Our detector has a high potential to detect microemboli up to now undetectable, but unfortunately it has a high potential to detect artifacts as well.

\section{CONCLusion}

The examination quality was improved. The new SLP allows us either to decrease the false alarm rate if all microemboli were detected, or to improve the microemboli detection with any false alarm.

\section{REFERENCES}

[1] M. Spencer, G. Thomas, S. Nicholls, and L. Sauvage, "Detection of middle cerebral artery emboli during carotid endarterectomy using transcranial doppler ultrasonography," Stroke, vol. 21, no. 3, pp. 415-423, 1990.

[2] J.-M. Girault, M. Biard, D. Kouame, A. Bleuzen, and F. Tranquart, "Spectral correlation of the embolic blood doppler signal," in Proc. IEEE International Conference on Acoustics, Speech and Signal Processing ICASSP 2006, vol. 2, May 14-19, 2006, p. II.

[3] J.-M. Girault, D. Kouame, A. Ouahabi, and F. Patat, "Micro-emboli detection: an ultrasound doppler signal processing viewpoint," Ieee Transactions On Biomedical Engineering, vol. 47, no. 11, pp. 1431-1439, nov 2000.

[4] E. V. VanZuilen, W. H. Mess, C. Jensen, I. VanDerTweel, J. VanGijn, and R. G. A. Ackerstaff, "Automatic embolus detection compared with human experts - a doppler ultrasound study," Stroke, vol. 27, no. 10, pp. 1840-1843, Oct. 1996.

[5] J.-M. Girault, M. Biard, D. Kouamé, G. Souchon, A. Bleuzen, and F. Tranquart, "Etude de la cyclostationnarité du signal doppler sanguin pour la détection de micro-emboles : étude préliminaire," in $20^{\circ}$ Colloque sur le traitement du signal et des images, 2005, pp. 206-209. 